\title{
ARTICLE \\ Overexpression of steroid receptor coactivators alleviates hyperglycemia-induced endothelial cell injury in rats through activating the PI3K/Akt pathway
}

\author{
Xiao-juan Quan ${ }^{1}$, Chun-lian Liang ${ }^{1}$, Ming-zhu Sun ${ }^{1}$, Lin Zhang ${ }^{1}$ and Xiu-li Li ${ }^{1}$
}

\begin{abstract}
Hyperglycemia is a major factor in vascular endothelial injury that finally leads to a cardiovascular event. Steroid receptor coactivators (SRCS) are a group of non-DNA binding proteins that induce structural changes in steroid receptors (nuclear receptors) critical for transcriptional activation. SRCs, namely, SRC-1, SRC-2, and SRC-3, are implicated in the regulation of vascular homeostasis. In this study we investigate the role of SRCs in hyperglycemia-induced endothelial injury. Aortic endothelial cells were prepared from normal and diabetic rats, respectively. Diabetic rats were prepared by injection of streptozotocin ( $50 \mathrm{mg} / \mathrm{kg}$, i.p.). The expression levels of SRC-1 and SRC-3 were significantly decreased in endothelial cells from the diabetic rats. Similar phenomenon was also observed in aortic endothelial cells from the normal rats treated with a high glucose $(25 \mathrm{mM})$ for $4 \mathrm{~h}$ or $8 \mathrm{~h}$. The expression levels of SRC-2 were little affected by hyperglycemia. Overexpression of SRC-1 and SRC-3 in high glucose-treated endothelial cells significantly increased the cell viability, suspended cell senescence, and inhibited cell apoptosis compared with the control cells. We further showed that overexpression of SRC-1 and SRC-3 markedly suppressed endothelial injury through restoring nitric oxide production, upregulating the expression of antioxidant enzymes (SOD, GPX, and CAT), and activating the PI3K/Akt pathway. The beneficial effects of SRC-1 and SRC-3 overexpression were blocked by treatment with the PI3K inhibitor LY294002 (10 mM) or with the Akt inhibitor MK-2206 (100 nM). In conclusion, hyperglycemia decreased SRC-1 and SRC-3 expression levels in rat aortic endothelial cells. SRC-1 and SRC-3 overexpression might protect against endothelial injury via inhibition of oxidative stress and activation of PI3K/Akt pathway.
\end{abstract}

Keywords: diabetics; streptozotocin; hyperglycemia; endothelial cells; steroid receptor coactivators; cell senescence; cell apoptosis; oxidative stress; PI3K/Akt pathway; LY294002; MK-2206

Acta Pharmacologica Sinica (2019) 40:648-657; https://doi.org/10.1038/s41401-018-0109-4

\section{INTRODUCTION}

It has been estimated that due to the growing prevalence, the number of patients with diabetes in China will increase to 42.3 million in 2030 [1]. This complex metabolic disorder is characterized by hyperglycemia and insulin resistance, which induce vascular stiffening and increase the susceptibility of the arterial wall to atherosclerosis in patients [2]. Consequently, severe pathological alterations to the arterial wall may enhance the occurrence of cardiovascular events, resulting in a significant burden to health care expenditure and increased mortality rates.

The vascular endothelium is now widely regarded as an active organ that plays a central role in the maintenance of vascular homeostasis. Studies have shown that the endothelium is involved in the control of thrombosis and thrombolysis, the interactions of platelets and leukocytes with the vessel wall, the regulation of vascular tone, and the growth of blood vessels [3]. However, the vascular endothelium is sensitive to the state of blood flow. For example, endothelial damage can be induced by diabetes-associated hyperglycemia [4, 5]. High glucose conditions could suppress endothelial nitric oxide synthase (eNOS) activity in endothelial cells by activating the hexosamine pathway and causing endothelial dysfunction [6]; moreover, through the influence of SIRT1 expression, hyperglycemia might trigger endothelial senescence, which could be antagonized by metformin treatment [7]. Furthermore, hyperglycemia could alter the function of endothelial cells via the activation of the calcium-dependent protease calpain and the $\mathrm{Na}^{+} / \mathrm{H}^{+}$exchanger [8].

The steroid receptor coactivators (SRCs) are a group of non-DNA binding proteins that induce structural changes in steroid receptors (or nuclear receptors) that are critical for transcriptional activation [9, 10]. The SRCs are comprised of three homologous members, i.e., SRC1, SRC-2, and SRC-3. These three proteins share several conserved domains, such as the basic helix loop helix-Per Arnt Sims (bHLH-PAS) motif in the N-terminus, which is crucial for protein-protein interactions, the nuclear receptor interaction domain (NRID) in the central region and the $A D 1$ and $A D 2$ domains in the C-terminal half. SRC-1 was the first identified nuclear receptor coactivator (also called nuclear receptor coactivator 1, NCOA1). SRC-2 is also named transcriptional intermediary factor-2 (TIF-2) and nuclear receptor coactivator 2 (NCOA2), and SRC-3 has been called nuclear receptor coactivator 3 (NCOA3), activator of thyroid and retinoic acid receptor

${ }^{1}$ Department of Geriatrics \& Endocrinology, The Second Affiliated Hospital of Xi'an Jiaotong University, Xi'an 710004, China

Correspondence: Xiao-juan Quan (xiaojuanquan@163.com)

Received: 13 November 2017 Accepted: 4 July 2018

Published online: 08 August 2018 
(ACTR), and thyroid hormone receptor activator molecule 1 (TRAM-1). All of these proteins have been shown to play important roles in transcription initiation, elongation, RNA splicing, receptor and coregulator turnover, and mRNA translation [11, 12]. Therefore, the ablation or overexpression of SRCs is directly linked to various disease outcomes. Many studies have reported their established roles in reproductive, developmental, and cancer biology [13]. SRCs are also implicated in the regulation of vascular homeostasis. SRC-1 is expressed in endothelial cells, vascular smooth muscle cells and neointima cells, and facilitates vasoprotection through inhibiting neointima formation after vascular injury [14]. The loss of SRC-3 expression in endothelial cells leads to the inhibition of proliferation and motility in response to growth factors and prevents the formation of intact monolayers with tight junctions in 2D culture and endothelial tubes in 3D culture [15]. Limited reports have investigated the role of SRC-2 in vascular biology and disease. Notably, SRC-2 acts as an indispensable integrator of transcriptional complexes that control the rate-limiting steps of hepatic glucose release and accretion [16]. SRC-2 is also involved in tumor cell metabolic reprogramming, orchestrating the circadian rhythm and maintaining the heart transcriptional profile [16-18]. Cyclin B1, cyclin D1, and cyclin E2 belong to a family that is critical for endothelial cell cycling [19]. In addition, dysfunctional phosphatidylinositol-4,5bisphosphate 3-kinase (PI3K)/protein kinase B (Akt) signaling has been shown to be responsible for the development of vascular complications in diabetes [20].

Until now, little has been published on the role of SRCs in endothelial injury caused by diabetes-associated hyperglycemia. In this study, we will explore the expression of SRCs in endothelial cells treated with a high glucose concentration and the effects of SRCs on endothelial injury.

\section{MATERIALS AND METHODS}

Diabetic rat model establishment

Six-week-old male Sprague-Dawley (SD) rats weighing 150-175 g were housed under controlled environmental conditions with an equal light-dark cycle. The animals were randomly divided into control and diabetic groups (10 in each group). A diabetic rat model was established with a single intraperitoneal injection of streptozotocin (STZ, 50 mg/kg, \#S0310, Sigma-Aldrich, St. Louis, MO, USA) in citrate buffer ( $\mathrm{pH} 4.4, \# C 2488$, Sigma-Aldrich). The control rats were injected with citrate buffer. Glucose levels in tail vein blood samples were determined using a glucose meter (ACCU-CHEK, Roche, Switzerland). Diabetes was confirmed by stable hyperglycemia (blood glucose levels of $250-350 \mathrm{mg} / \mathrm{dl}$ ) two weeks after STZ injection. Six weeks after STZ injection, the rats were sacrificed with an overdose of anesthesia (intraperitoneal injection of pentobarbital sodium, $50 \mathrm{mg} / \mathrm{kg}$ ); then, fasting blood glucose and insulin levels were determined. The current study was approved by the Animal Ethical Committee of Xi'an Jiaotong University (No. XJTU2AF2017LS112, April 2017).

Rat aortic endothelial cell isolation

Rat aortic endothelial cells were isolated from control and diabetic SD rats according to a previous study [21]. Briefly, SD rats were anesthetized with an injection of pentobarbital sodium $(50 \mathrm{mg} / \mathrm{kg}$, intraperitoneally). The rat aortas were removed and cleaned using sterilized saline. The vessels were cut along the axial direction and then cut into small pieces $\left(<2 \mathrm{~mm}^{2}\right)$. Next, the tissue pieces were washed and resuspended in $1 \mathrm{~mL}$ of Eagle's MEM (\#11965-092, Gibco) containing $20 \%$ fetal calf serum before they were placed into 24-well plates $(0.4 \mathrm{~mL}$ in each well) coated with gelatin. The minced tissues were incubated at $37{ }^{\circ} \mathrm{C}$ with $5 \% \mathrm{CO}_{2}$ for $3-4$ days, and the cells grown from the tissues were harvested. Rat aortic endothelial cells were identified with anti-rat factor VIII and FITC-conjugated rabbit anti-goat lgG antibodies. The cells were cultured in Eagle's MEM (\#11965-092, Gibco) containing 10\% FBS (\#13011-8611,
Zhejiang Tianhang Biotechnology, China), 1\% penicillinstreptomycin and $0.15 \mathrm{mg} / \mathrm{mL}$ EC growth supplement at $37^{\circ} \mathrm{C}$ in a $5 \% \mathrm{CO}_{2}$ incubator. In addition, $0.1 \%$ gelatin-coated $60-\mathrm{mm}$ culture dishes were used for endothelial cell culture. The mRNA and protein expression levels of SRC-1, SRC-2, and SRC-3 in these primary endothelial cells were analyzed. For the experiments performed on normal endothelial cells, the cells from the primary culture at passage 3 were used. The cells were incubated with either $5.5 \mathrm{mM}$ D-glucose (normal) or $25 \mathrm{mM} \mathrm{D}$-glucose as indicated for $4 \mathrm{~h}$ or $8 \mathrm{~h}$. To exclude data variability resulting from the different osmotic pressures in the normal and high glucose groups, $19.5 \mathrm{mM}$ mannitol was added to the normal group as an osmotic control. To inhibit ROS production, endothelial cells were pre-incubated with $10 \mathrm{mM} \mathrm{N}$ acetyl-L-cysteine (NAC, \#0108, Amresco, Solon, OH, USA) for $2 \mathrm{~h}$. To inhibit PI3K activity, endothelial cells were pre-treated with $10 \mu \mathrm{M}$ LY294002 (\#9901, Cell Signaling Tech, Beverly, MA, USA) for 2 h. To inhibit Akt activity, endothelial cells were incubated with $100 \mathrm{nM}$ MK-2206 for $2 \mathrm{~h}$ before high glucose exposure.

Plasmid construction and transfection

The full-length human SRC-1 (NC 000002.12) and SRC-3 (NC_000020.11) genes were cloned and then inserted into the EcoRI/BamHI sites of a pcDNA3.1 vector. For plasmid transfection, endothelial cells were plated in 6-well dishes and grown to $60 \%$ confluency. Then, the cells were transfected with the pcDNA3.1-SRC1 and/or pcDNA3.1-SRC-3 plasmids using LipoFiter Liposomal Transfection Reagent (\#HB-Tr-1000, Hanbio Biotechnology, Shanghai, China) according to the manufacturer's instructions. Western blots were used to assess the transfection efficacy.

\section{Cell viability detection}

Endothelial cell viability was evaluated using 3-(4,5-dimethyl-2thiazolyl)-2,5-diphenyl-2- $H$-tetrazolium bromide (MTT) assays. Cells were placed in 96-well plates $\left(1 \times 10^{5}\right.$ cells $\left./ \mathrm{mL}\right)$ and treated with a high glucose concentration ( $25 \mathrm{mM}$ D-glucose) for $0.5 \mathrm{~h}, 1 \mathrm{~h}, 4 \mathrm{~h}$, and $8 \mathrm{~h}$. The medium was then removed and changed to normal Eagle's MEM with $10 \%$ FBS for another $24 \mathrm{~h}$ of culture. Cell viability assays were carried out according to the manufacturer's protocol (\#ST316, Beyotime, Jiangsu, China). After incubation for $4 \mathrm{~h}$, DMSO $(150 \mu \mathrm{L} /$ well) was added to end the reaction, and the absorbance was determined at the indicated time with an ELISA plate reader at 570 $\mathrm{nm}$.

\section{Cell senescence detection}

Cell senescence was detected using a Senescence $\beta$-Galactosidase Staining Kit according to the manufacturer's instructions (\#C0602, Beyotime Biotechnology, China). SA- $\beta$-Gal-stained cells were quantified by counting 500 cells per dish, and images were taken using a phase-contrast microscope at $\times 400$ magnification (Olympus, Tokyo, Japan). The proportion of cells positive for SA- $\beta$-Gal activity is shown as the percentage of the total number of cells counted in each dish.

\section{Apoptosis analysis}

Endothelial cell apoptosis was assessed using an Annexin V-FITC Propidium lodide (PI) Apoptosis Detection Kit (\#40302ES20, Yeasen, Shanghai, China). Cells $\left(2 \times 10^{5}\right.$ cells $\left./ \mathrm{mL}\right)$ were incubated with trypsinEDTA, washed twice with PBS, and resuspended in $1 \mathrm{~mL}$ of Annexin V binding buffer. Next, Annexin V-FITC and PI were added to the medium $\left(5 \mathrm{~mL} / 10^{5}\right.$ cells). The samples were mixed gently, incubated for $15 \mathrm{~min}$ at room temperature in the dark, and then subjected to flow cytometry to evaluate the number of apoptotic cells.

\section{Antioxidant enzymes}

The levels of antioxidant enzymes, including superoxide dismutase (SOD, \#S0109, Beyotime Biotechnology), glutathione peroxidase (GPX, \#S0056, Beyotime Biotechnology), and catalase (CAT, \#S0051, Beyotime Biotechnology), expressed by endothelial cells were 
examined using biochemical analysis kits. The experiments were repeated at least three times.

Nitric oxide production

The Griess method was employed to determine nitric oxide (NO) production in endothelial cells overexpressing SRC- 1 and SRC-3 or treated with NAC. Briefly, endothelial cells were incubated with $10 \mathrm{U} / \mathrm{mL}$ nitrate reductase and $5 \mathrm{mM}$ nicotinamide adenine dinucleotide phosphate oxidase at room temperature for $3 \mathrm{~h}$. Next, these cells were treated with Griess reagent (\#K262, BioVision, Milpitas, CA, USA) for $20 \mathrm{~min}$. Lastly, the absorbances of all the samples were determined with a microplate reader at $540 \mathrm{~nm}$.

\section{qRT-PCR analysis}

Total RNA from endothelial cells was extracted and reverse transcribed into first-strand cDNA. The target genes were normalized to the internal reference $\beta$-actin, and all analyses were performed on a 7300 Real-Time PCR system (ABI). PCR was carried out with SYBR Green Premix Taq (\#RR420L, Takara, Dalian, China) and $40 \mathrm{ng}$ of template CDNA after incubation with uracil- $N$-glycosylase for 2 min at $50^{\circ} \mathrm{C}$. Denaturation was performed at $95^{\circ} \mathrm{C}$ for $10 \mathrm{~min}$, followed by 40 cycles of denaturation at $95^{\circ} \mathrm{C}$ for $30 \mathrm{~s}$, annealing at $55^{\circ} \mathrm{C}$ for $30 \mathrm{~s}$ and extension at $72{ }^{\circ} \mathrm{C}$ for $60 \mathrm{~s}$. All reactions were performed in triplicate. $\beta$-Actin was employed as an internal control. $\mathrm{Ct}$ values were obtained from triplicate samples and averaged. The $\Delta \mathrm{Ct}$

Table 1. Detection of blood glucose level and insulin level in Sprague-Dawley rats

\begin{tabular}{lll}
\hline Groups & Fasting blood glucose $(\mathrm{mM})$ & Fasting insulin $(\mathrm{mU} / \mathrm{L})$ \\
\hline Normal controls & $5.4 \pm 0.5$ & $7.2 \pm 0.8$ \\
Diabetic rats & $19.6 \pm 2.3^{*}$ & $4.1 \pm 0.3^{*}$ \\
\hline Mean \pm SD, $n=10, t$-test & \\
${ }^{*} P<0.05$ vs. normal controls & \\
\hline
\end{tabular}

indicates the difference between the mean $\mathrm{Ct}$ for the specific cDNAs. The $\Delta \Delta \mathrm{Ct}$ indicates the average $\Delta \mathrm{Ct}$ for an indicated treatment minus the average $\Delta \mathrm{Ct}$ of the control samples. The data are expressed as relative quantifications and calculated as $2^{-\Delta \Delta C t}$. The primers used for qRT-PCR were as follows: SRC-1 (accession: NM_001108012.1), forward 5'-TACCAGCGTCTACAGCAT-3', reverse 5'-AGCATTGTCCCATCATTC-3'; SRC'-2 (accession: NM_031822.1), forward 5'-CGGAACAGCCATACCTTC-3', reverse 5'-GCTTATCTTCGC CCTCCT-3'; and SRC-3 (accession: NM_181659.2), forward 5'AGCAGAGGATGGCGATGA-3', reverse 5'-GGAAACTGTTGTGGAGG-3'.

Western blotting

Endothelial cells were homogenized and lysed in RIPA buffer. Whole cell extract preparations were obtained, and the protein concentrations were determined using BCA protein assays. Forty micrograms of protein was separated on 10\% (w/v) SDS-PAGE gels (Beyotime) and transferred onto PVDF membranes (\#1620176, Bio-Rad, Hercules, CA, USA). After blocking with 5\% (w/v) BSA (\#A3858, Sigma-Aldrich) and washing with Tris-buffered saline with Tween buffer, the membranes were incubated overnight at $4{ }^{\circ} \mathrm{C}$ with primary antibodies. The primary antibodies included anti-SRC-1 (1:800, \#2191, Cell Signaling), anti-SRC-2 (1:1000, \#96687, Cell Signaling), anti-SRC-3 (1:800, \#2126, Cell Signaling), anti-cyclin B1(1:1000, \#12231, Cell Signaling), anticyclin D1 (1:1000, \#2978, Cell Signaling), anti-cyclin E2 (1:1000, \#20808, Cell Signaling), anti-PI3K (1:1000, \#4249, Cell Signaling), anti-phosphoPI3K (1:1000, \#13857, Cell Signaling), anti-Akt (1:800, \#4691, Cell Signaling), anti-phospho-Akt (1:1000, \#4060, Cell Signaling), anti-Bcl-2 (1:1000, \#2764, Cell Signaling), anti-Bax (1:1000, \#5023, Cell Signaling), anti-phospho-eNOS (1:1000, \#9574, Cell Signaling), anti-eNOS (1:1000, \#9572, Cell Signaling), and anti- $\beta$-actin (1:800, \#3700, Cell Signaling). Following this incubation, HRP-conjugated secondary antibody (1:10000, \#7074, Cell Signaling) was added, and the protein bands were visualized by incubating the membranes with chemiluminescence reagent prior to exposure to a gel imaging system (GE Healthcare). The protein band intensities of the target genes were normalized to that of $\beta$-actin in the same gel lane.


Fig. 1 Steroid receptor coactivator (SRC; SRC-1, SRC-2, and SRC-3) expression levels in normal and diabetic rats. a SRC mRNA expression levels in aortic endothelial cells isolated from normal and diabetic rats. $\mathbf{b}$ SRC protein expression levels in aortic endothelial cells. Mean \pm SD, $n=6$. $t$-test, ${ }^{*} P<$ 0.05 vs. normal controls 
a
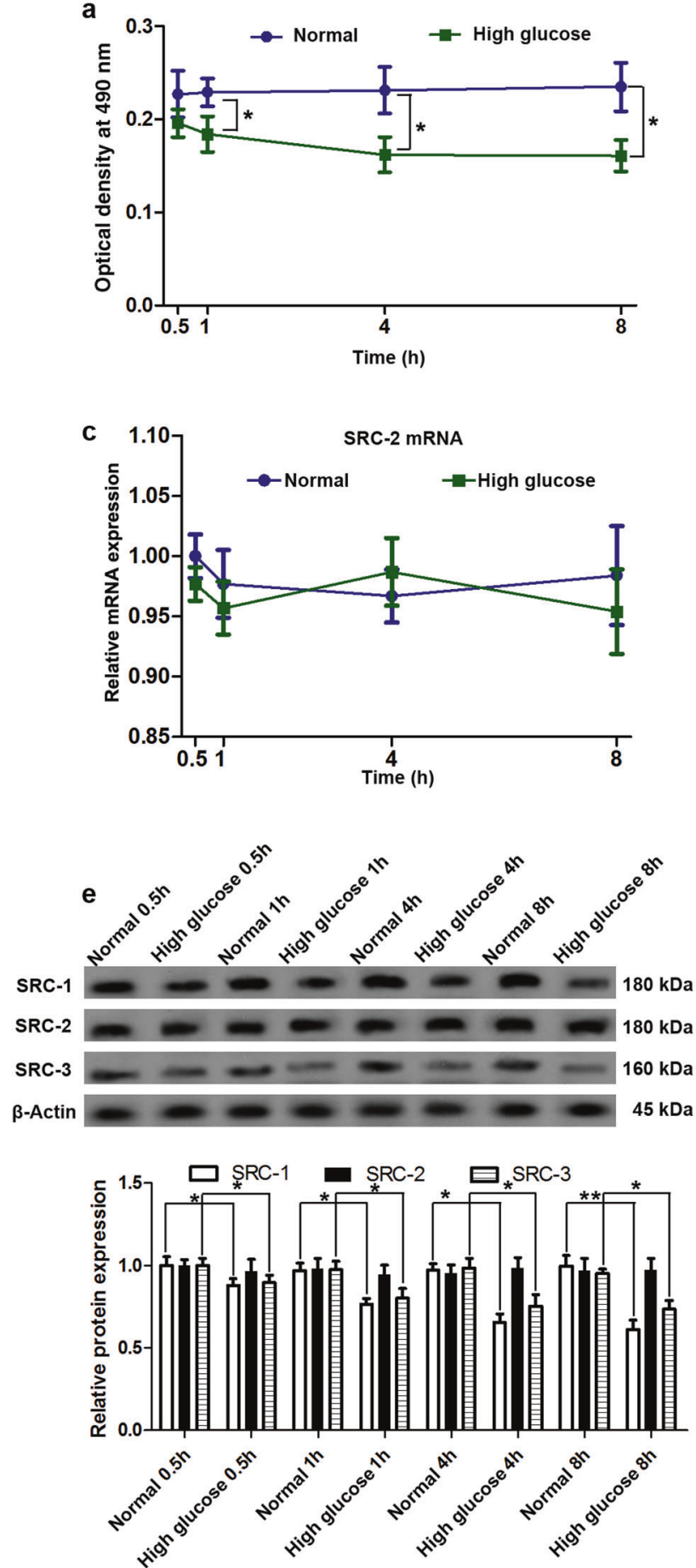


Fig. 2 Steroid receptor coactivator (SRC; SRC-1, SRC-2, and SRC-3) expression levels in rat aortic endothelial cells treated with a normal or high glucose concentration. a Cell survival after normal or high glucose treatment for $0.5 \mathrm{~h}, 1 \mathrm{~h}, 4 \mathrm{~h}$, or $8 \mathrm{~h}$. b SRC-1, c SRC-2, d SRC-3 mRNA expression levels in aortic endothelial cells with normal or high glucose treatment for $0.5 \mathrm{~h}, 1 \mathrm{~h}, 4 \mathrm{~h}$, or $8 \mathrm{~h}$. e SRC protein expression levels in aortic endothelial cells with normal or high glucose treatment for $0.5 \mathrm{~h}, 1 \mathrm{~h}, 4 \mathrm{~h}$, or $8 \mathrm{~h}$. $\mathrm{f}$ Cyclin B1, cyclin D1, and cyclin E2 expression levels in aortic endothelial cells. Mean $\pm S D, n=6$. Double factor variance analysis, ${ }^{*} P<0.05,{ }^{* *} P<0.01$

Statistical analysis

All data are presented as the mean \pm SD. Statistical analyses were performed using one-way ANOVA followed by Dunnett's multiple comparison test (SPSS 17.0, Chicago, IL, USA). Comparisons between two groups were analyzed using Student's $t$-test. $P<0.05$ was considered statistically significant.

\section{RESULTS}

SRC-1 and SRC-3 expression is downregulated in the aortic endothelium of diabetic rat models

To explore the effects of hyperglycemia on the expression of SRC-1, SRC-2, and SRC-3, a rat model of diabetes was established by streptozotocin injection. Six weeks after the last injection, the rats 



Fig. 3 Effects of SRC-1 and SRC-3 overexpression on rat aortic endothelial cells with high glucose treatment. a SRC-1 and SRC-3 expression levels after plasmid transfection. b Cell survival, c cell senescence (400x), and d apoptosis with SRC-1 and SRC-3 overexpression and high glucose treatment; I indicates the control group, II indicates the high glucose group, III indicates the blank group, IV indicates the SRC-1 overexpression group, $\mathrm{V}$ indicates the SRC-3 overexpression group, and $\mathrm{VI}$ indicates the SRC- $1+\mathrm{SRC}-3$ overexpression group, scale bar $=15$ $\mu \mathrm{m}$. Mean $\pm \mathrm{SD}, n=6$. One-way ANOVA, ${ }^{*} P<0.05$ vs. control, ${ }^{\#} P<0.05$ vs. high glucose

were sacrificed with an anesthesia overdose. Subsequently, blood glucose and insulin levels were determined, and aortic endothelial cells were isolated from the intima of the rat aortas. Our data show that the blood glucose levels were significantly higher in diabetic rats than in control rats, while the insulin levels were much lower (Table 1). qRT-PCR and Western blot analyses were employed to evaluate the expression of SRC-1, SRC-2, and SRC-3 in aortic endothelial cells isolated from diabetic and control rats. The results show that the expression levels of SRC-1 and SRC-3 were significantly lower in diabetic rats than in control rats, while the expression levels of SRC-2 remained similar (Fig. 1a, b). Our results suggest that hyperglycemia might decrease the expression levels of SRC- 1 and SRC-3 in the aortic endothelium but have little effect on SRC-2 expression.

High glucose treatment reduces SRC-1 and SRC-3 expression levels in endothelial cells

To confirm the effects of high glucose treatment on endothelial cells, rat aortic endothelial cells were exposed to glucose (normal: $5.5 \mathrm{mM}$ with $19.5 \mathrm{mM}$ mannitol; high: $25 \mathrm{mM}$ ) for $0.5 \mathrm{~h}, 1 \mathrm{~h}, 4 \mathrm{~h}$, and $8 \mathrm{~h}$. At the end of the treatment period, endothelial cell survival was evaluated. The results of the MTT assays showed that high glucose exposure reduced endothelial cell survival (Fig. 2a). 
a


b



c
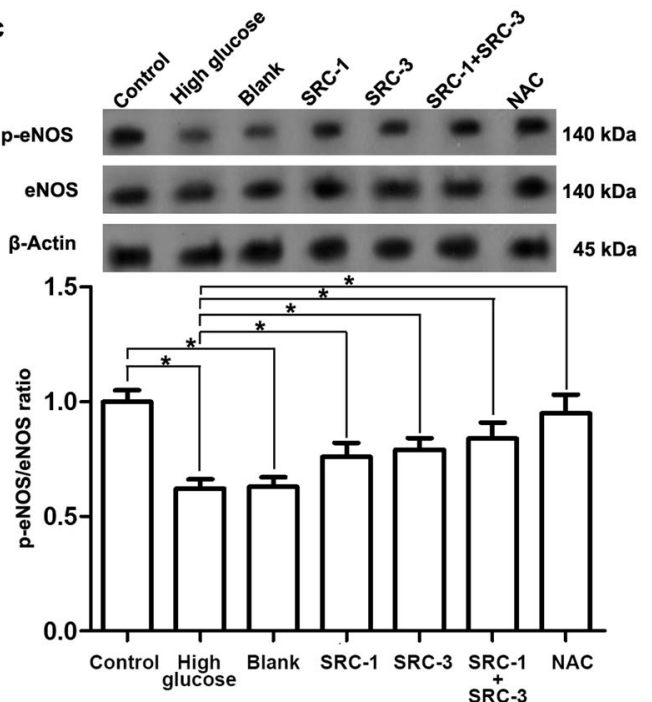

d

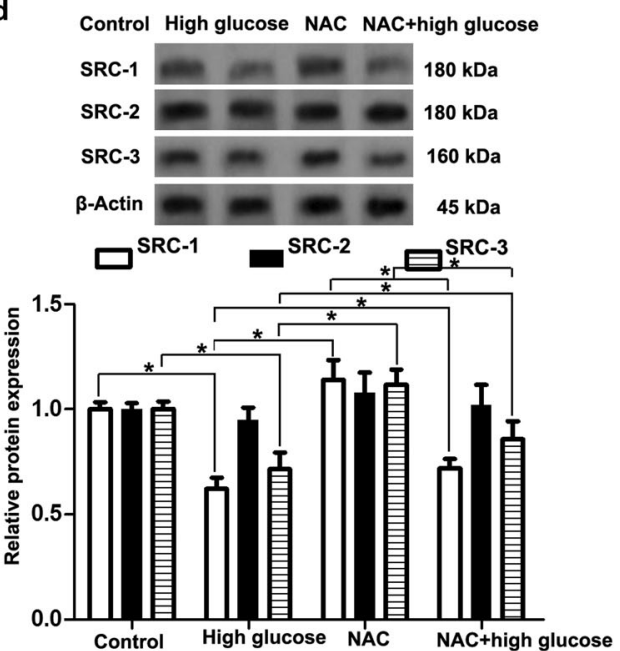

e

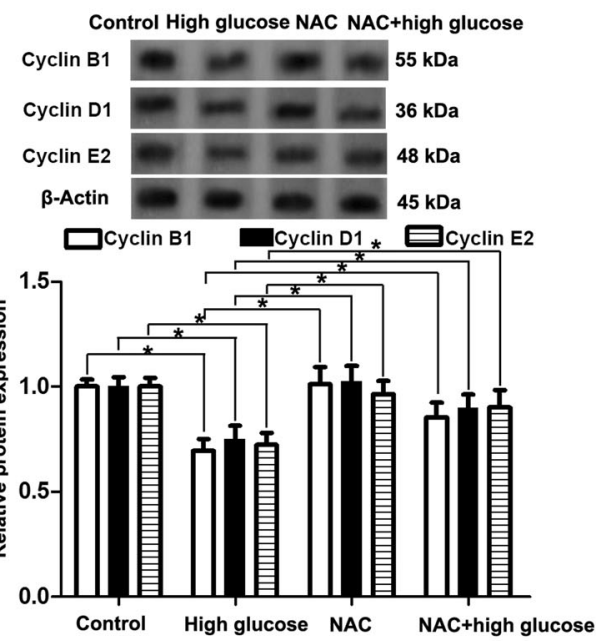

f
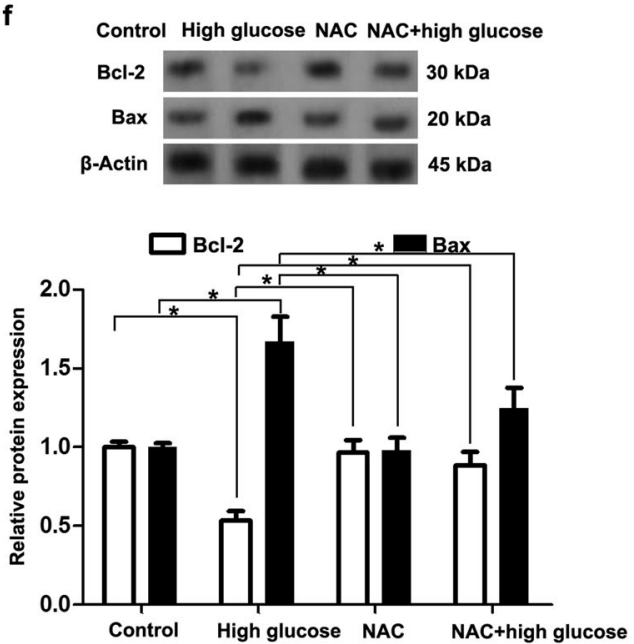

Fig. 4 Activation of the PI3K/Akt pathway in rat aortic endothelial cells with SRC-1 and SRC-3 overexpression. a Analysis of PI3K, p-PI3K, Akt and p-Akt expression levels in endothelial cells, $n=6$. b Measurement of NO production in endothelial cells with SRC- 1 and SRC-3 overexpression, $n=10$. c Analysis of p-eNOS and eNOS expression levels in endothelial cells, $n=10$. d Effects of NAC on SRC-1, SRC-2, and SRC-3 expression levels, $n=6$. e Effects of NAC on cyclin B1, cyclin D1, and cyclin E2 expression levels, $n=6$. $\mathbf{f}$ Effects of NAC on Bcl-2 and Bax expression levels, $n=6$. Mean \pm SD. One-way ANOVA, ${ }^{*} P<0.05$ 
Table 2. Evaluation of the activity of the antioxidant enzymes

\begin{tabular}{llll}
\hline Groups & SOD $(\mathrm{U} / \mathrm{mL})$ & $\mathrm{GPX}(\mathrm{U} / \mathrm{mL})$ & $\mathrm{CAT}(\mathrm{U} / \mathrm{mL})$ \\
\hline Control & $0.54 \pm 0.03$ & $0.63 \pm 0.08$ & $0.24 \pm 0.03$ \\
High glucose & $0.42 \pm 0.06^{*}$ & $0.35 \pm 0.04^{*}$ & $0.09 \pm 0.01^{*}$ \\
Blank & $0.41 \pm 0.05^{*}$ & $0.36 \pm 0.03^{*}$ & $0.11 \pm 0.01^{*}$ \\
SRC-1 overexpression & $0.51 \pm 0.06^{\#}$ & $0.46 \pm 0.07^{* \#}$ & $0.19 \pm 0.02^{* \#}$ \\
SRC-3 overexpression & $0.49 \pm 0.05^{\#}$ & $0.49 \pm 0.08^{* \#}$ & $0.18 \pm 0.01^{* \#}$ \\
SRC-1+SRC-3 overexpression & $0.52 \pm 0.04^{\#}$ & $0.45 \pm 0.07^{* \#}$ & $0.19 \pm 0.02^{* \#}$ \\
NAC & $0.53 \pm 0.05^{\#}$ & $0.64 \pm 0.09^{\#}$ & $0.26 \pm 0.04^{\#}$ \\
\hline Mean \pm SD, $n=10$, one-way ANOVA & & \\
${ }^{*} P<0.05$ vs. control, ${ }^{*} P<0.05$ vs. high glucose & & \\
SOD superoxide dismutase, GPX glutathione peroxidase, CAT catalase
\end{tabular}

Furthermore, the mRNA and protein expression levels of SRC-1, SRC-2, and SRC-3 were also determined. The results show that compared with those in the control group, SRC- 1 and SRC- 3 mRNA levels were decreased at $1 \mathrm{~h}, 4 \mathrm{~h}$, and $8 \mathrm{~h}$, with only slight changes in SRC-2 mRNA expression levels (Fig. 2b, c, d). In line with the mRNA expression data, SRC-1 and SRC-3 protein expression levels were lower in the high glucose group than in the control group, whereas SRC-2 protein expression levels did not change significantly (Fig. 2e). Previous studies have indicated that SRC-1 and SRC-3 participate in the regulation of cyclin B1, cyclin D1, and cyclin E2 $[22,23]$. Therefore, the expression of cyclin B1, cyclin D1, and cyclin E2 was explored in endothelial cells after glucose exposure. Our results show that the expression levels of cyclin B1, cyclin D1, and cyclin E2 were reduced with high glucose treatment compared with control treatment (Fig. 2f). These results suggest that high glucose levels might inhibit SRC-1 and SRC-3 expression in endothelial cells in vitro.

\section{SRC-1 and SRC-3 modulate high glucose treatment-induced endothelial cell survival, senescence, and apoptosis}

To confirm the effects of SRC- 1 and SRC-3 on endothelial cells, fulllength SRC-1 and SRC-3 vectors were constructed and transfected into rat aortic endothelial cells (Fig. 3a); then, the cells were treated with glucose. The MTT assay results show that endothelial cell viability was enhanced with SRC-1 and/or SRC-3 overexpression (Fig. 3b). To assess endothelial cell senescence, senescenceassociated $\beta$-Galactosidase was analyzed using a staining kit. Our data show that high glucose treatment resulted in cell senescence, while the overexpression of SRC-1 and/or SRC-3 attenuated the endothelial cell senescence induced by high glucose treatment (Fig. 3c). Furthermore, endothelial cell apoptosis was analyzed by flow cytometry. Higher percentages of annexin $\mathrm{V}^{+} \mathrm{PI}^{+}$and annexin $\mathrm{V}^{+} \mathrm{PI}^{-}$endothelial cells were induced by high glucose treatment than control treatment (Fig. 3d). In contrast, the overexpression of SRC-1 and/or SRC-3 decreased the proportion of apoptotic endothelial cells (Fig. 3d). In sum, our results reveal that SRC-1 and SRC-3 overexpression offset the effects of high glucose treatment on endothelial cells.

SRC- 1 and SRC-3 promote the PI3K/Akt signaling suppressed by high glucose treatment

To further elucidate the roles of SRC- 1 and SRC- 3 in the protection of endothelial cells following high glucose treatment, the activation of the PI3K/Akt pathway was examined in endothelial cells. Compared with control treatment, high glucose treatment suppressed the expression of phosphorylated PI3K (p-PI3K) and pAkt (Fig. 4a). Nonetheless, the overexpression of SRC-1 and/or SRC3 enhanced $\mathrm{p}$-PI3K and $\mathrm{p}$-Akt expression in endothelial cells (Fig. 4a). Furthermore, oxidative stress has been reported to mediate the effects of high glucose treatment on endothelial cells and to dampen PI3K/Akt signaling. In SRC-1 and/or SRC-3 overexpressing cells, augmented activity levels of the antioxidant enzymes SOD, GPX, and CAT were detected (Table 2). SRC-1 and SRC-3 overexpression also recovered NO production and the $\mathrm{p}$ eNOS/eNOS ratio in endothelial cells (Figs. 4b, c). Additionally, NAC, which was used to inhibit oxidative stress, upregulated the expression of p-PI3K and p-Akt in endothelial cells (Fig. 4a, Table 2). In addition, NAC treatment significantly enhanced SRC-1, SRC-3, cyclin, and $\mathrm{BCl}-2$ expression, as well as NO production and the $p$-eNOS/eNOS ratio, whereas NAC decreased the Bax expression levels induced by high glucose treatment (Fig. 4b, $c$, d, e, f). Additionally, LY294002 and MK-2206 were used to inhibit PI3K activation and Akt activation, respectively. We found that LY294002 and MK-2206 reduced cell viability, enhanced cell senescence and promoted apoptosis in endothelial cells with SRC1 and SRC-3 overexpression (Fig. 5a, b, c). Moreover, LY294002 and MK-2206 significantly suppressed NO production and eNOS expression in endothelial cells with SRC- 1 and SRC-3 overexpression (Fig. 5d, e). Collectively, our results reveal that SRC-1 and SRC-3 attenuate endothelial cell injury by suppressing oxidative stress and activating the PI3K/Akt pathway.

\section{DISCUSSION}

Hyperglycemia occurs in patients with type 1 or type 2 diabetes mellitus and constitutes the major reason for cardiovascular damage due to its activation of the protein kinase $C$, polyol, and hexosamine pathways, as well as the production of advanced glycation end products [24]. High glucose conditions can further cause mitochondrial dysfunction and endoplasmic reticulum stress, inducing ROS production and promoting cellular injury $[25,26]$. Aortic endothelial cell dysfunction, inflammation, and death are commonly observed in hyperglycemia-associated complications [27, 28]. Although many studies have reported the role of hyperglycemia in endothelial cell injury, the molecular mechanism remains to be explained. In our study, we characterized the expression of three transcriptional coactivators, i.e., SRC-1, SRC-2, and SRC-3, in a rat model of diabetes. The expression levels of SRC- 1 and SRC-3 were decreased in the rat aortic endothelium, with only a slight change in the expression of SRC-2, suggesting that hyperglycemia might have an impact on the rat aorta and affect SRC- 1 and SRC-3 expression in the aortic endothelium (Fig. 1). In vitro, high glucose treatment reduced endothelial cell survival and dampened SRC-1 and SRC-3 expression but did not influence SRC-2 expression (Fig. 2). In parallel, the expression of cyclin B1, cyclin D1, and cyclin E2 in endothelial cells was also downregulated by high glucose treatment (Fig. 2). These results reveal the modulatory effect of high glucose levels on SRC expression and endothelial cell survival. It is not clear why the expression of SRC-2 was not affected by hyperglycemia in vivo and in vitro. A previous study has shown that SRC-2 acts as a nuclear receptor coactivator as well as a corepressor [29]. We speculate that the distinct functions of the SRC-2 gene may depend on not only its expression but also its interaction with other transcription factors. High glucose exposure may alter the interactions of SRC-2 in endothelial cells.

Previous studies have demonstrated the tight association between SRC-1 and SRC-3 and the maintenance of normal vascular function. The deletion of SRC-1 has been reported to cause high blood pressure and increase aortic stiffness in mice [30]. In vascular smooth muscle cells, SRC-1 mediates the regulation of inflammatory genes following angiotensin II treatment and is responsible for IL-6 expression [31]. Likewise, SRC-3, which is highly expressed in vascular smooth muscle cells and endothelial cells, plays a role in the estrogen-mediated inhibition of neointimal growth and the protection of vascular function [32]. Furthermore, SRC-3 is involved in regulating endothelial cell migration, proliferation, and tube formation by upregulating 


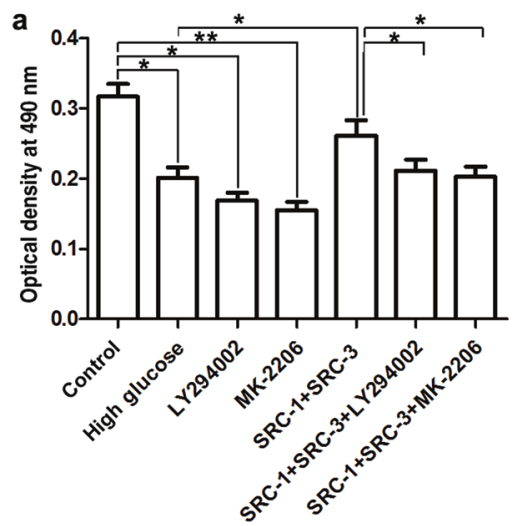

b
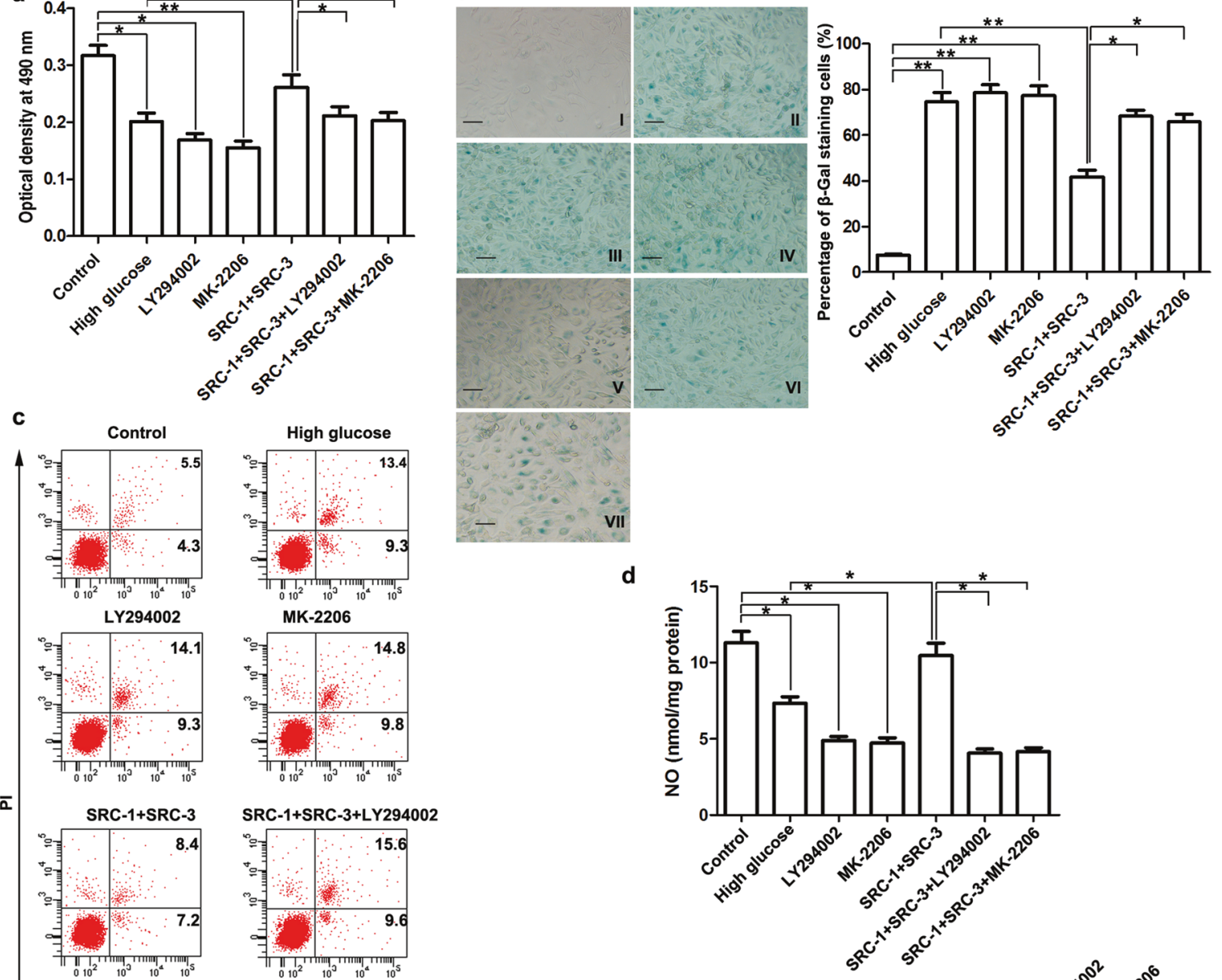

SRC-1+SRC-3+MK-2206


d
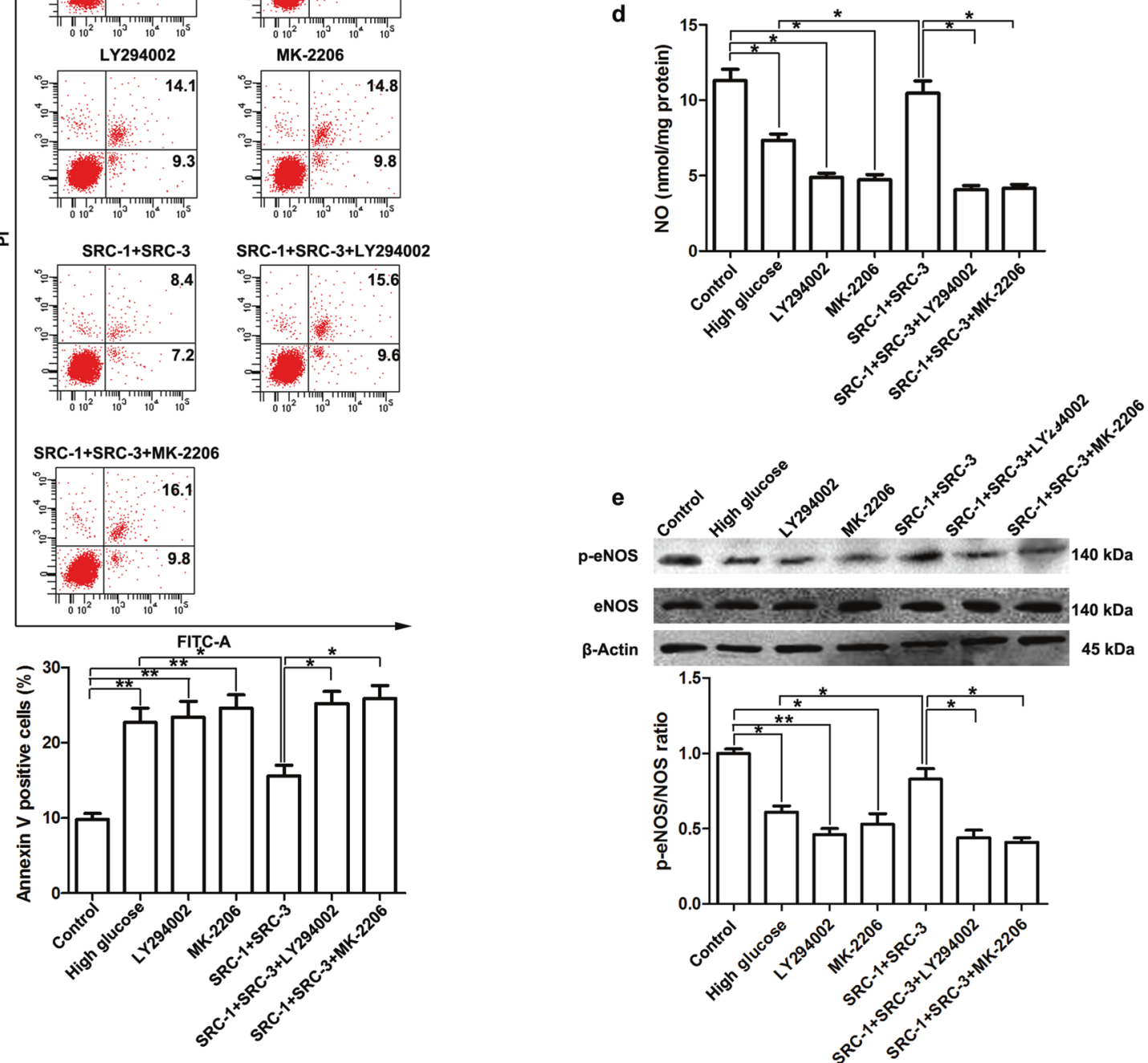

Fig. 5 Inhibition of the PI3K/Akt pathway counters the effects of SRC-1 and SRC-3 overexpression. a Cell survival, $n=10, \mathbf{b}$ cell senescence $(\times 400), n=6$, and c apoptosis in aortic endothelial cells treated with LY294002 (10 mM, $2 \mathrm{~h}$ ) or MK-2206 (100 nM, $2 \mathrm{~h}$ ), $n=6$. d NO production $(n=10)$ and p-eNOS/NOS expression levels $(n=6)$ in aortic endothelial cells treated with LY294002 $(10 \mathrm{mM}, 2 \mathrm{~h})$ or MK-2206 $(100 \mathrm{nM}, 2 \mathrm{~h})$ were determined. I indicates the control group, II indicates the high glucose group, III indicates the LY294002 group, IV indicates the MK-2206 group, V indicates the SRC-1 + SRC-3 overexpression group, VI indicates the SRC-1 + SRC-3 + LY294002 group, and VII indicates the SRC-1 + $\mathrm{SRC}-3+$ MK-2206 group, scale bar $=15 \mu \mathrm{m}$. Mean \pm SD. One-way ANOVA, ${ }^{*} P<0.05,{ }^{* *} P<0.01$ 
VEGFR2 expression [33]. In this study, SRC-1 and SRC-3 increased cell viability, attenuated cell senescence, and decreased apoptosis in aortic endothelial cells following high glucose treatment (Fig. 3). Thus, our study demonstrates that SRC- 1 and SRC-3 could protect endothelial cells from high glucose treatment-induced damage. SRC- 1 has been shown to interact with HIF-1a and enhance its transactivation potential in a hypoxia-dependent manner [34]. Hence, the overexpression of SRC- 1 and SRC-3 might counteract endothelial cell injury induced by high glucose treatment through the activation of a series of downstream signals.

The current study shows that the overexpression of SRC- 1 and SRC-3 upregulated p-PI3K and p-Akt expression, which was suppressed by high glucose treatment. The activation of PI3K/ Akt signaling has been shown to prevent apoptosis, promote proliferation, and reverse dysfunction in endothelial cells [35, 36]. Therefore, increased SRC-1 and SRC-3 levels might protect endothelial cells by stimulating PI3K/Akt signaling. Oxidative stress plays a role in high glucose treatment-induced endothelial dysfunction and the development of a pro-inflammatory phenotype $[37,38]$, which play important roles in micro- and macrovascular diseases. Interestingly, our study shows the restoration of NO production in high glucose-treated cells with SRC-1 and SRC-3 overexpression; this restoration also promoted the expression of antioxidant enzymes, including SOD, GPX, and CAT (Fig. 4, Table 2). Because SRC-1 and SRC-3 are coactivators of steroid receptors, including the receptors for estrogen, progesterone, and glucocorticoids, we suspect that the modulation of these antioxidant enzymes might be attributed to their interaction with steroid receptors. The generation of superoxide in endothelial cells under high glucose conditions contributes to pathological alterations, such as NO synthesis inhibition, vascular inflammation, and remodeling [24]. Hyperglycemia can cause the dysregulation of some essential proteins associated with oxidative stress; these proteins include NAD(P)H oxidase, SIRT1, and p-eNOS/eNOS [7, 39, 40]. In this study, exposure to high glucose conditions decreased the p-eNOS/eNOS ratio in aortic endothelial cells, while the overexpression of SRC- 1 and SRC-3 recovered the p-eNOS/eNOS ratio; these data reveal the role of SRC-1 and SRC-3 in the suppression of superoxide generation (Fig. 4c). Moreover, we also noted that the alleviation of oxidative stress with NAC affected the expression levels of SRC-1 and SRC-3 (Fig. 4d), as well as cyclins and apoptotic proteins (Fig. 4e, f). The results suggested that oxidative stress can, in turn, impact SRC- 1 and SRC-3 expression and endothelial cell survival under high glucose conditions.

In summary, this study provides evidence that high glucose conditions cause aortic endothelial cell injury through the downregulation of SRC-1 and SRC-3 expression and the suppression of the PI3K/Akt pathway. The overexpression of SRC- 1 and SRC-3 enhances cell viability and attenuates cell injury. This study emphasizes the role of SRC- 1 and SRC-3 in the endothelial damage induced by hyperglycemia and might help to understand the mechanisms responsible for vascular lesions in diabetes. SRC-1 and SRC-3 are potential targets for diabetes treatment.

\section{ACKNOWLEDGEMENTS}

Financial support was provided by the Key Program for Social Development, Science and Technology of Shaanxi Province, China (2016SF-077), and the Social Development Guidance Program-Medical Research Project of Xi'an, China (SF1509).

\section{AUTHOR CONTRIBUTIONS}

$\mathrm{XJQ}$ and CLL designed research and wrote the paper; XJQ, CLL, MZS, LZ, and XLL performed research; and XJQ, CLL, LZ, and XLL analyzed data.

\section{ADDITIONAL INFORMATION}

Conflict of interest: The authors declare that they have no conflict of interest.
Publisher's note: Springer Nature remains neutral with regard to jurisdictional claims in published maps and institutional affiliations.

\section{REFERENCES}


mellitus among inland residents in China (2000-2014): a meta-analysis. J Diabetes Investig. 2016;7:845-52.

2. Kozakova M, Palombo C. Diabetes mellitus, arterial wall, and cardiovascular risk assessment. Int J Environ Res Public Health. 2016;13:201.

3. Rajendran P, Rengarajan T, Thangavel J, Nishigaki Y, Sakthisekaran D, Sethi G, et al. The vascular endothelium and human diseases. Int J Biol Sci. 2013;9:1057-69.

4. Hoffman RP. Vascular endothelial dysfunction and nutritional compounds in early type 1 diabetes. Curr Diabetes Rev. 2014;10:201-7.

5. Paneni F, Beckman JA, Creager MA, Cosentino F. Diabetes and vascular disease: pathophysiology, clinical consequences, and medical therapy: part I. Eur Heart J. 2013;34:2436-43.

6. Du XL, Edelstein D, Dimmeler S, Ju Q, Sui C, Brownlee M. Hyperglycemia inhibits endothelial nitric oxide synthase activity by posttranslational modification at the Akt site. J Clin Invest. 2001;108:1341-8.

7. Arunachalam G, Samuel SM, Marei I, Ding H, Triggle CR. Metformin modulates hyperglycaemia-induced endothelial senescence and apoptosis through SIRT1. Br J Pharmacol. 2014;171:523-35.

8. Wang $\mathrm{S}$, Peng $\mathrm{Q}$, Zhang J, Liu L. $\mathrm{Na}+/ \mathrm{H}+$ exchanger is required for hyperglycaemia-induced endothelial dysfunction via calcium-dependent calpain. Cardiovasc Res. 2008;80:255-62.

9. Stashi E, York B, O'Malley BW. Steroid receptor coactivators: servants and masters for control of systems metabolism. Trends Endocrinol Metab. 2014;25:337-47.

10. Johnson AB, O'Malley BW. Steroid receptor coactivators 1, 2, and 3: critical regulators of nuclear receptor activity and steroid receptor modulator (SRM)-based cancer therapy. Mol Cell Endocrinol. 2012;348:430-9.

11. York B, O'Malley BW. Steroid receptor coactivator (SRC) family: masters of systems biology. J Biol Chem. 2010;285:38743-50.

12. Leo C, Chen JD. The SRC family of nuclear receptor coactivators. Gene. 2000:245:1-11.

13. Walsh CA, Qin L, Tien JC, Young LS, Xu J. The function of steroid receptor coactivator-1 in normal tissues and cancer. Int J Biol Sci. 2012;8:470-85.

14. Yuan $Y, X u$ J. Loss-of-function deletion of the steroid receptor coactivator-1 gene in mice reduces estrogen effect on the vascular injury response. Arterioscler Thromb Vasc Biol. 2007;27:1521-7.

15. Ying $\mathrm{H}$, Willingham $M C$, Cheng SY. The steroid receptor coactivator-3 is a tumor promoter in a mouse model of thyroid cancer. Oncogene. 2008;27:823-30.

16. Fleet $T$, Zhang B, Lin F, Zhu B, Dasgupta S, Stashi E, et al. SRC-2 orchestrates polygenic inputs for fine-tuning glucose homeostasis. Proc Natl Acad Sci USA. 2015;112:E6068-6077.

17. Stashi E, Lanz RB, Mao J, Michailidis G, Zhu B, Kettner NM, et al. SRC-2 is an essential coactivator for orchestrating metabolism and circadian rhythm. Cell Rep. 2014;6:633-45.

18. Reineke EL, York B, Stashi E, Chen X, Tsimelzon A, Xu J, et al. SRC-2 coactivator deficiency decreases functional reserve in response to pressure overload of mouse heart. PLoS ONE. 2012;7:e53395.

19. Sherr CJ, Roberts JM. Living with or without cyclins and cyclin-dependent kinases. Genes Dev. 2004;18:2699-711.

20. Nie XQ, Chen HH, Zhang JY, Zhang YJ, Yang JW, Pan HJ, et al. Rutaecarpine ameliorates hyperlipidemia and hyperglycemia in fat-fed, streptozotocin-treated rats via regulating the IRS-1/PI3K/Akt and AMPK/ACC2 signaling pathways. Acta Pharmacol Sin. 2016;37:483-96.

21. McGuire PG, Orkin RW. Isolation of rat aortic endothelial cells by primary explant techniques and their phenotypic modulation by defined substrata. Lab Invest. 1987:57:94-105.

22. Chen X, Qin L, Liu Z, Liao L, Martin JF, Xu J. Knockout of SRC-1 and SRC-3 in mice decreases cardiomyocyte proliferation and causes a noncompaction cardiomyopathy phenotype. Int J Biol Sci. 2015;11:1056-72.

23. Gonzalez-Arenas A, Hansberg-Pastor V, Hernandez-Hernandez OT, GonzalezGarcia TK, Henderson-Villalpando J, Lemus-Hernandez D, et al. Estradiol increases cell growth in human astrocytoma cell lines through ERalpha activation and its interaction with SRC-1 and SRC-3 coactivators. Biochim Biophys Acta. 2012;1823:379-86.

24. Fiorentino TV, Prioletta A, Zuo P, Folli F. Hyperglycemia-induced oxidative stress and its role in diabetes mellitus related cardiovascular diseases. Curr Pharm Des. 2013;19:5695-703.

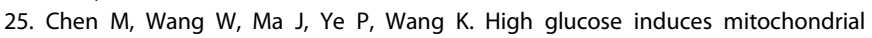
dysfunction and apoptosis in human retinal pigment epithelium cells via promoting SOCS1 and Fas/FasL signaling. Cytokine. 2016;78:94-102. 
26. Dassanayaka S, Readnower RD, Salabei JK, Long BW, Aird AL, Zheng YT, et al. High glucose induces mitochondrial dysfunction independently of protein OGlcNAcylation. Biochem J. 2015;467:115-26.

27. Wu N, Shen H, Liu H, Wang Y, Bai Y, Han P. Acute blood glucose fluctuation enhances rat aorta endothelial cell apoptosis, oxidative stress and proinflammatory cytokine expression in vivo. Cardiovasc Diabetol. 2016;15:109.

28. Cifarelli V, Lee S, Kim DH, Zhang T, Kamagate A, Slusher S, et al. FOXO1 mediates the autocrine effect of endothelin-1 on endothelial cell survival. Mol Endocrinol. 2012;26:1213-24.

29. Cvoro A, Tzagarakis-Foster C, Tatomer D, Paruthiyil S, Fox MS, Leitman DC. Distinct roles of unliganded and liganded estrogen receptors in transcriptional repression. Mol Cell. 2006;21:555-64.

30. Hinton $A O J$, Yang $Y$, Quick $A P, X u P$, Reddy $C L$, Yan $X$, et al. SRC-1 regulates blood pressure and aortic stiffness in female mice. PLoS ONE. 2016;11: e0168644.

31. Sahar S, Reddy MA, Wong C, Meng L, Wang M, Natarajan R. Cooperation of SRC-1 and p300 with NF-kappaB and CREB in angiotensin II-induced IL-6 expression in vascular smooth muscle cells. Arterioscler Thromb Vasc Biol. 2007;27: 1528-34.

32. Yuan Y, Liao L, Tulis DA, Xu J. Steroid receptor coactivator-3 is required for inhibition of neointima formation by estrogen. Circulation. 2002;105:2653-9.

33. Wang W, Bian K, Vallabhaneni S, Zhang B, Wu RC, O'Malley BW, et al. ERK3 promotes endothelial cell functions by upregulating SRC-3/SP1-mediated VEGFR2 expression. J Cell Physiol. 2014;229:1529-37.
34. Carrero P, Okamoto K, Coumailleau P, O'Brien S, Tanaka H, Poellinger L. Redox regulated recruitment of the transcriptional coactivators CREB-binding protein and SRC-1 to hypoxia-inducible factor 1alpha. Mol Cell Biol. 2000;20:402-15.

35. Zeng L, Xiao Q, Chen M, Margariti A, Martin D, Ivetic A, et al. Vascular endothelial cell growth-activated XBP1 splicing in endothelial cells is crucial for angiogenesis. Circulation. 2013;127:1712-22.

36. Li J, Xiong J, Yang B, Zhou Q, Wu Y, Luo H, et al. Endothelial cell apoptosis induces TGF-beta signaling-dependent host endothelial-mesenchymal transition to promote transplant arteriosclerosis. Am J Transplant. 2015;15:3095-111.

37. Karbach S, Jansen T, Horke S, Heeren T, Scholz A, Coldewey M, et al. Hyperglycemia and oxidative stress in cultured endothelial cells--a comparison of primary endothelial cells with an immortalized endothelial cell line. J Diabetes Complicat. 2012;26:155-62.

38. Brouwers O, Niessen PM, Haenen G, Miyata T, Brownlee M, Stehouwer CD, et al. Hyperglycaemia-induced impairment of endothelium-dependent vasorelaxation in rat mesenteric arteries is mediated by intracellular methylglyoxal levels in a pathway dependent on oxidative stress. Diabetologia. 2010;53:989-1000.

39. Xi G, Shen X, Wai C, Vilas CK, Clemmons DR. Hyperglycemia stimulates p62/ PKCzeta interaction, which mediates NF-kappaB activation, increased Nox4 expression, and inflammatory cytokine activation in vascular smooth muscle. FASEB J. 2015;29:4772-82.

40. Wang W, Wang WH, Azadzoi KM, Dai P, Wang Q, Sun JB, et al. Alu RNA accumulation in hyperglycemia augments oxidative stress and impairs eNOS and SOD2 expression in endothelial cells. Mol Cell Endocrinol. 2016;426:91-100. 